\title{
The Influence of Benzophenone Substitution on the Physico-Chemical Characterizations of 8-HydroxyQuinoline NLO Single Crystals
}

\author{
M. J. Jarald Brigit Gilda ${ }^{1}$, S. Anbarasu ${ }^{2}$, Y. Samson ${ }^{3}$, Prem Anand Devarajan ${ }^{2 *}$ \\ ${ }^{1}$ Department of Physics, Ponjesly College of Engineering, Alamparai, India \\ ${ }^{2}$ Deparment of Physics, St. Xavier's College, Palayamkottai, India \\ ${ }^{3}$ Departmentof Physics, Annai Velankanni College, Tholayavattam, India \\ Email: *d.prenanand2010@gmail.com
}

Received February 12, 2012; revised March 20, 2012; accepted April 7, 2012

\begin{abstract}
Single crystals of 8-HydroxyQuinoline (8-HQ) and Benzophenone substituted 8-HydroxyQuinoline (B8-HQ) are grown by slow evaporation of acetone at room temperature. Coloured crystals of 8-HQ and B8-HQ with good optical quality of dimensions $54 \times 3 \times 1.5 \mathrm{~mm}^{3}$ and $27 \times 3 \times 1 \mathrm{~mm}^{3}$ are harvested. Single crystal X-ray diffractometer was utilized to measure the unit cell parameters and to confirm the crystal structure. The presence of various functional groups in the molecule was ascertained by FTIR spectral analysis. The cut off wavelength of 8-HQ and B8-HQ was centered at 350 and $356 \mathrm{~nm}$. The functional groups in the molecule are elucidated by ${ }^{1} \mathrm{H}$ and ${ }^{13} \mathrm{C}$-NMR spectral analyses. Kurtz Perry test confirms the SHG in8-HQ andB8-HQ single crystals.
\end{abstract}

Keywords: Crystal Growth; X-Ray Diffraction (XRD); FTIR; UV-Vis-NIR; ${ }^{1} \mathrm{H}$ and ${ }^{13} \mathrm{C}-\mathrm{NMRe}$

\section{Introduction}

Nonlinear optical materials (NLO) have wide applications in the area of laser technology, optical communication and in storage devices. The high non linearity makes it possible for organic crystals to double the frequency of Ga-Al-As diode lasers for generating blue light which is an important coherent source [1]. One of the advantages in working with organic materials is that they allow one to fine-tune the chemical structures and properties for the desired nonlinear optical properties. In addition, organic crystals have large optical susceptibility, inherent ultrafast response and high optical threshold for laser power as compared with inorganic materials. Moreover, their lower cutoff wavelength and a wide transparency window in the visible region makes the candidate materials subject for extensive investigation [2].

In this context 8-HydroxyQuinoline (8-HQ) has been identified as an important material for NLO applications in the visible and ultraviolet regions. M. Rajasekaran has reported the growth of 8-HQ single crystal by slow evaporation technique using chloroform as a solvent. The cut off wavelength is below $400 \mathrm{~nm}$ [3]. Recently K. Aravinth has reported that the cut off wavelength of $8-\mathrm{HQ}$ is around $390 \mathrm{~nm}$ and the lattice parameters are $\mathrm{a}=$

${ }^{*}$ Corresponding author.
$3.85 \AA, b=24.93 \AA, c=28.72 \AA, V=2754 \AA$. The grown 8-HQ crystal belongs to orthorhombic system with non-centro-symmetric space group Fdd2 [4]. Elena M. Filip et al. have performed quantum chemical semi empirical PM3 calculations optimization of 8-HydroxyQuinoline (8-HQ) structure, dipolemoment, polarizability and other physical parameters [5].

The sonochemical fabrication of 8-HydroxyQuinoline (8-HQ) aluminium $\left(\mathrm{Alq}_{3}\right)$ nanoflowers with high electro generated chemiluminescence was reported by Chang-Jie Mao et al. [6]. Due to the continuation of interest in the supramolecular arrangements, a new crystal structure of hydrated 8-HydroxyQuinoline chloride was synthesized from a reaction mixture consisting of 2-chloro nicotinoyl chloride and 8-HydroxyQuinoline (8-HQ) [7]. Additionally the tunable photoluminescence from tris (8-HQ) aluminium $\left(\mathrm{Alq}_{3}\right)$ blended thin films of $\mathrm{Alq}_{3}$ embedded in poly methyl methacrylate matrix at different concentrations are also investigated by J. G. Mahakhode et al. [8]. The interaction between donor 8-HQ and $\pi$ acceptor P-Nitrophenol has been investigated spectroscopically. It was shown by ${ }^{1} \mathrm{H}-\mathrm{NMR}$ that 8 -HQ with $\pi$ PNP forms complex of 1:1 stoichiometry. Infrared spectral data indicates a charge transfer interaction between donor and acceptor due to $\mathrm{n}-\pi^{*}$ transitions by the formation of radical ion pairs [9]. 
In this paper, we report the results of our work on the influence of physico chemical characterization studies of benzophenone substituted 8-HydroxyQuinoline (B8-HQ) NLO crystals along with characterization by single crystal X-ray diffraction (XRD), Fourier transform infrared (FTIR), UV-Visible-NIR and ${ }^{1} \mathrm{H} \&{ }^{13} \mathrm{C}$-Nuclear magnetic resonance (NMR) spectral measurements and NLO test for the first time.

\section{Experimental Details}

The starting materials are commercially available (EMerck). The solvent evaporation technique was used to grow single crystals of 8-HQ. The corresponding 8-HQ salt was dissolved in acetone to prepare a saturated solution and the solution was filtered using Whatmann filter paper. The filtered solution was then taken in a beaker which was hermetically sealed to avoid the evaporation of the solvent. Optically defect free good quality single crystals of 8-HQ with reasonable dimensions were harvested in a month. Benzophenone (5 M) was substituted with the solution containing 8-HQ and acetone. The concentration of the growth solution was fixed at $3 \mathrm{M}$. Within a month, seed crystals are formed due to spontaneous nucleation. In the present work, good optical quality crystals of pure 8-HQ and Benzophenone substituted 8-HQ crystals (B8-HQ) are harvested. Figures $\mathbf{1}$ and 2 show the photographs of asgrown pure 8-HQ and B8-HQ crystals.

\section{Single Crystal XRD}

The X-ray diffraction data was collected using an automatic X-ray diffractometer, EnrafNonius CAD 4-MV 31 Bruker kappa APE XIII. The structure was solved by the direct method using SHELXL program. It is observed from the X-ray diffraction data that both pure 8-HQ and

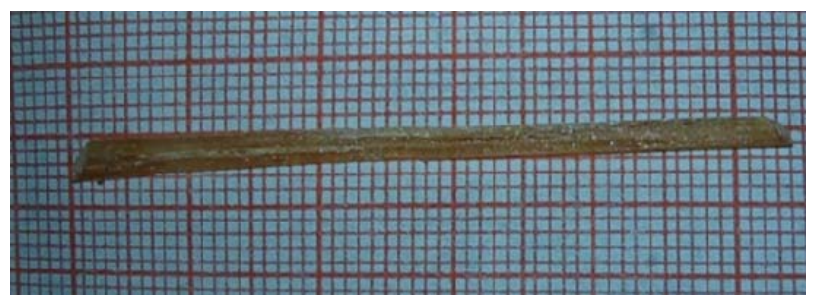

Figure 1. Photograph of as grown pure 8-HQ single crystal.

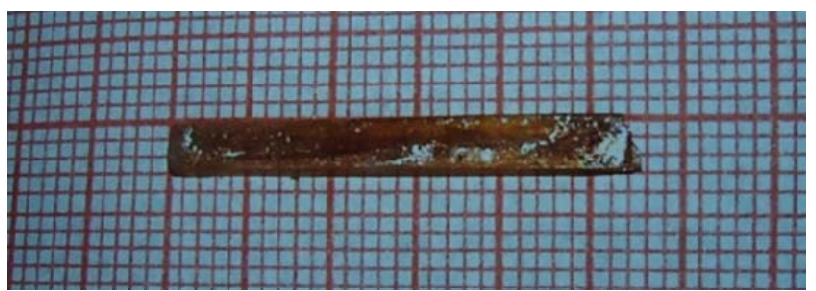

Figure 2. Photograph of as grown B8-HQ single crystal.
B8-HQ crystals are orthorhombic with a space group of Fdd2. The calculated lattice parameter values of pure 8-HQ and B8-HQ are presented in Table 1. The results of the present work are in good agreement with the reported values [4]. It is evident from Table 1, that the doped sample has a slight variation in the cell volume. The variation in the cell volume may be due to the incorporation of Benzophenone into 8-HQ crystal lattice.

\section{Ftir Spectral Analysis}

The FTIR spectroscopy study is effectively used to identify the functional groups present in the crystal and to determine the molecular structure. In order to analyze qualitatively the presence of the functional groups in 8-HQ and B8-HQ, FTIR was recorded using the Perkin Elmer Model spectrum-100 in the range $450-4000 \mathrm{~cm}^{-1}$ and the corresponding spectra obtained are shown in Figures 3 and $\mathbf{4}$ respectively. The FTIR spectrum of pure 8-HQ is presented in Figure 5. The aromatic $\mathrm{C}-\mathrm{H}$ stretching appears at $3029 \mathrm{~cm}^{-1}$. The band assigned at $1576 \mathrm{~cm}^{-1}$ is attributed to $\mathrm{C}=\mathrm{C}$ ring stretching vibrations. The $\mathrm{C}=\mathrm{N}$ stretching vibrations appears at the region of $1694 \mathrm{~cm}^{-1}$.

The peak at $3743 \mathrm{~cm}^{-1}$ is designated to $\mathrm{O}-\mathrm{H}$ stretching. The peaks at 1271 and $1222 \mathrm{~cm}^{-1}$ are assigned to $\mathrm{C}-\mathrm{O}$ stretching vibration. The aromatic $\mathrm{C}-\mathrm{H}$ plane bending appears at $1163 \mathrm{~cm}^{-1}$. The $\mathrm{C}-\mathrm{H}$ out of plane bending occurs at 707 and $738 \mathrm{~cm}^{-1}$. The peak at $1433 \mathrm{~cm}^{-1}$ is assigned to $\mathrm{O}-\mathrm{H}$ plane bending. The spectrum of B8-HQ is shown in Figure 4. The spectrum carries nearly similar features of pure 8-HQ spectra. An additional peak at $3057 \mathrm{~cm}^{-1}$ is observed which is due to the $\mathrm{C}=\mathrm{O}$ stretching of Benzophenone. An interesting observation to be mentioned here is all the peaks of Figure $\mathbf{4}$ are shifted to a higher frequencies of $1 \mathrm{~cm}^{-1}$, and hence it can be stated that there is harmonious existence of dopant Benzophenone into the crystal lattice of 8-HQ. Higher resolution of peaks and enhancement of the bands between the region $3400-1500 \mathrm{~cm}^{-1}$ are also observed.

Table 1. XRD data for 8-HQ and B8-HQ single crystal.

\begin{tabular}{ccc}
\hline Data & 8 -HQ & B8-HQ \\
\hline $\mathrm{a}(\AA)$ & 3.84 & 3.85 \\
$\mathrm{~b}(\AA)$ & 24.97 & 24.98 \\
$\mathrm{c}(\AA)$ & 28.68 & 28.71 \\
$\alpha^{\circ}$ & $90^{\circ}$ & $90^{\circ}$ \\
$\beta^{\circ}$ & $90^{\circ}$ & $90^{\circ}$ \\
$\gamma^{\circ}$ & $90^{\circ}$ & $90^{\circ}$ \\
Crystal system & Orthorhombic & Orthorhombic \\
Volume $(\AA)$ & 2749.97 & 2761.12 \\
Space group & Fdd2 & Fdd2 \\
\hline
\end{tabular}




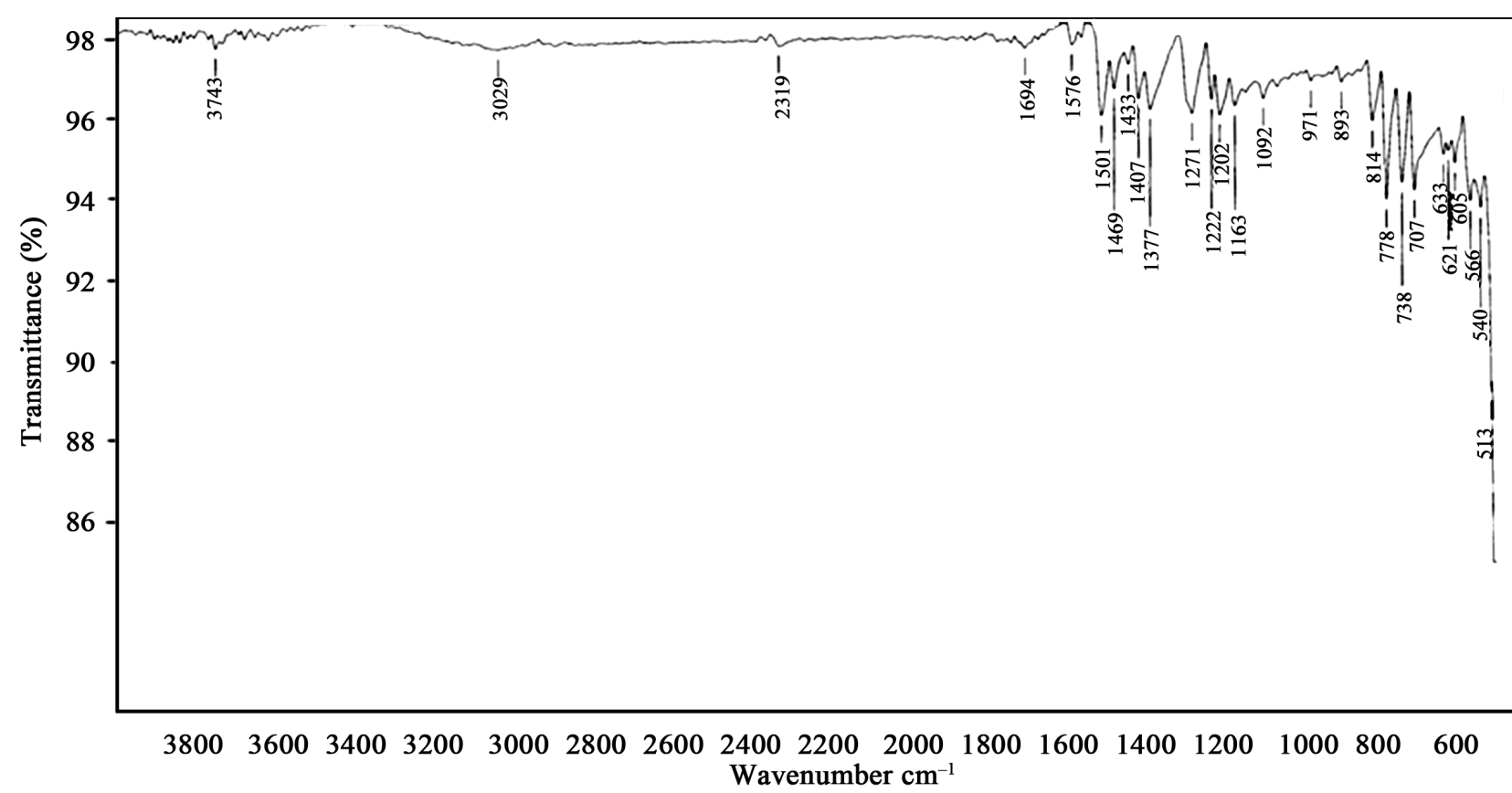

Figure 3. FTIR spectrum of as grown pure 8-HQ single crystal.

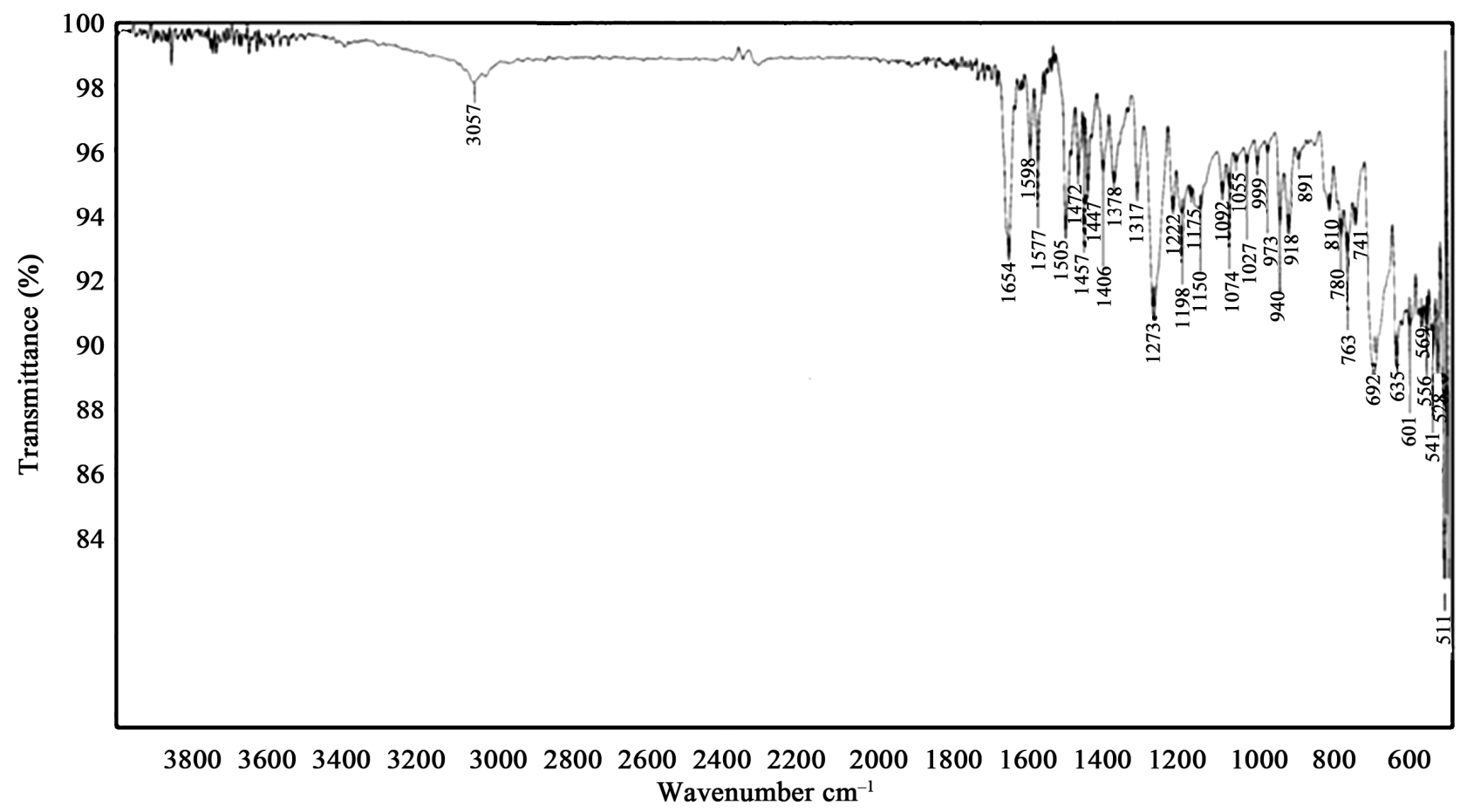

Figure 4. FTIR spectrum of as grown B8-HQ single crystal.

\section{UV-Vis-NIR Studies}

The UV-Visible absorption spectrum of pure 8-HQ and B8-HQ was recorded in the wavelength region $200-$ $1500 \mathrm{~nm}$ using a VARIAN CARY 5E model spectrophotometer and the obtained spectra are shown in Figure 6. When the absorbance is monitored from longer to shorter wavelength, the absorption is found to be moderately low in the visible region and the near infrared region of the spectrum. This is the most desirable property of materials possessing NLO activity. The cut off wavelength of 8HQ was found to be at $350 \mathrm{~nm}$ which is in agreement with reported values $[3,4]$ and the cut off wavelength of B8-HQ crystals was centered at $356 \mathrm{~nm}$ which is a desirable property for SONLO materials. 


\section{6. ${ }^{1} \mathrm{H}$ and ${ }^{13} \mathrm{C}-\mathrm{NMR}$ Spectral Analyses}

The ${ }^{1} \mathrm{H}$ NMR spectra of 8-HQ and B8-HQ were measured in Tetramethylsilane using the instrument Bruker AV III NMR spectrometer. ${ }^{1} \mathrm{H}$ and ${ }^{13} \mathrm{C}$ spectra of pure 8-HQ and B8-HQ are shown in Figures 5 and 7-9 respectively. The structure of 8 -HQ single crystal is

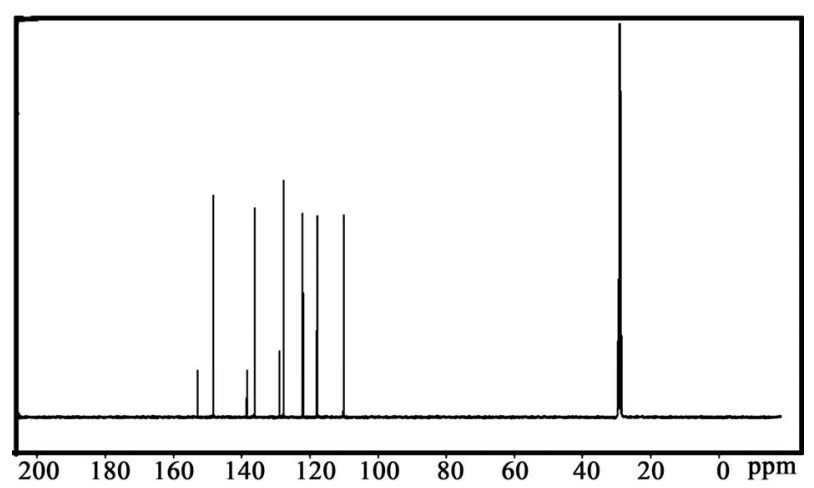

Figure $5 .{ }^{13} \mathrm{C}$ NMR spectrum of as grown B8-HQ single crystal.

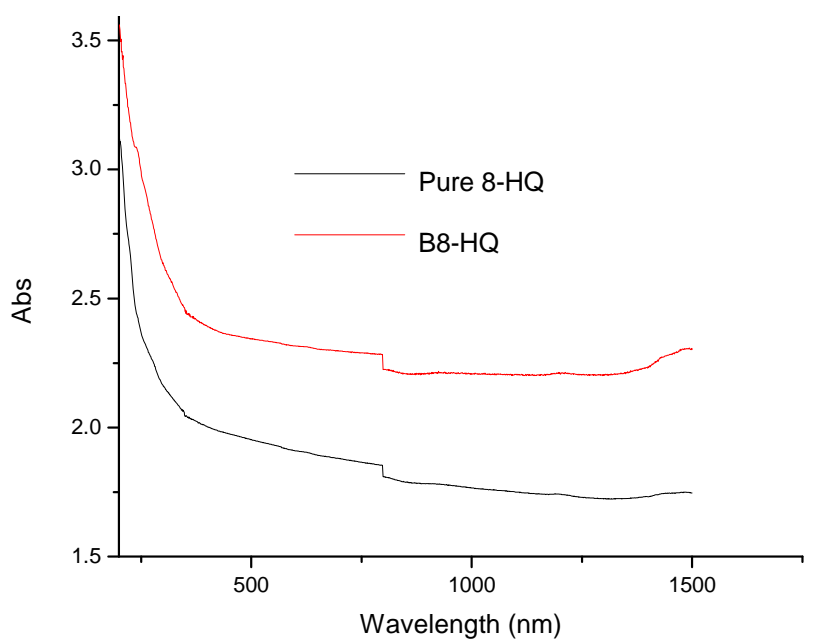

Figure 6. UV-Vis-NIR spectrum of as grown 8-HQ and B8-HQ single crystal.

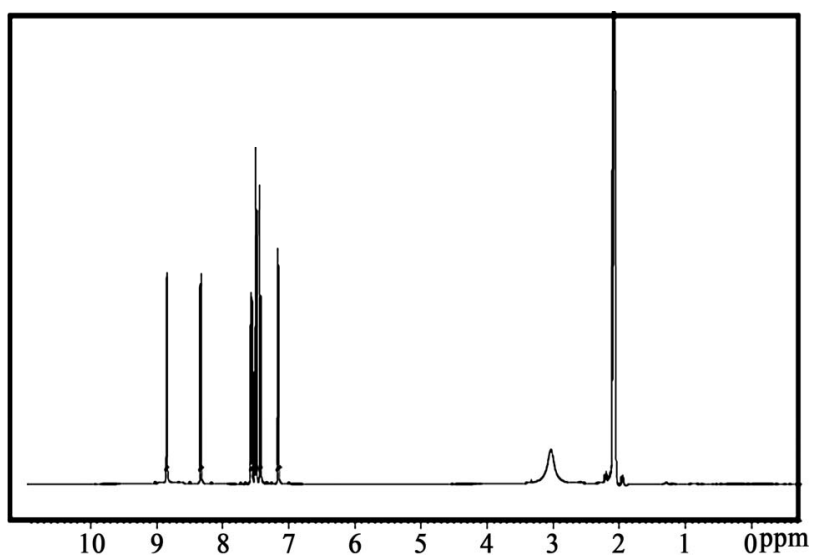

Figure 7. ${ }^{1} \mathrm{H}$ NMR spectrum of as grown 8-HQ single crystal. shown in Figure 10. A slight variation in ${ }^{1} \mathrm{H}-\mathrm{NMR}$ spectra is observed in the Benzophenone substituted spectra of 8-HQ. The addition of peaks centered around 7.175 and $7.160 \mathrm{ppm}$ may be attributed to the incorporation of Benzophenone in the 8-HQ crystal lattice. Thus it is clear from the spectra that the characteristic functional groups of 8-HQ and B8-HQ are evident. In the ${ }^{13} \mathrm{C}-\mathrm{NMR}$ spectra, the various functional groups in the carbon bonded network of 8-HQ and B8-HQ are shown in Figures 5 and $\mathbf{9}$ respectively. A slight variation is observed in the B8-HQ spectra. Thus it is ascertained from Figure 5, that the addition of ketone functional group is revealed in the spectrum.

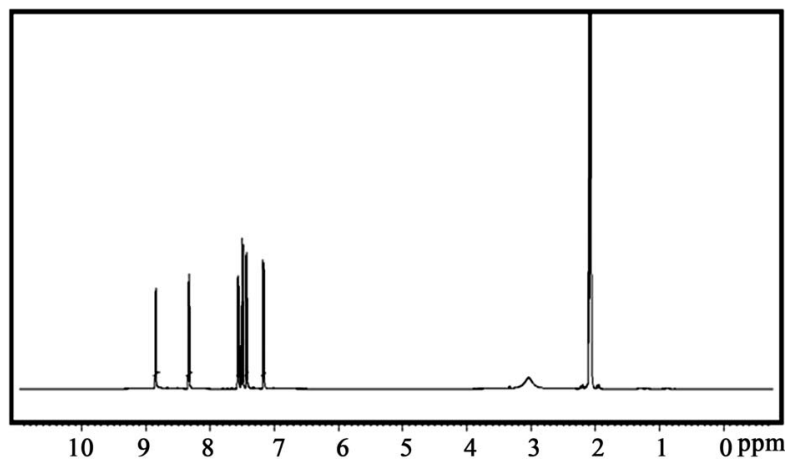

Figure 8. ${ }^{1} \mathrm{H}$ NMR spectrum of as grown B8-HQ single crystal.

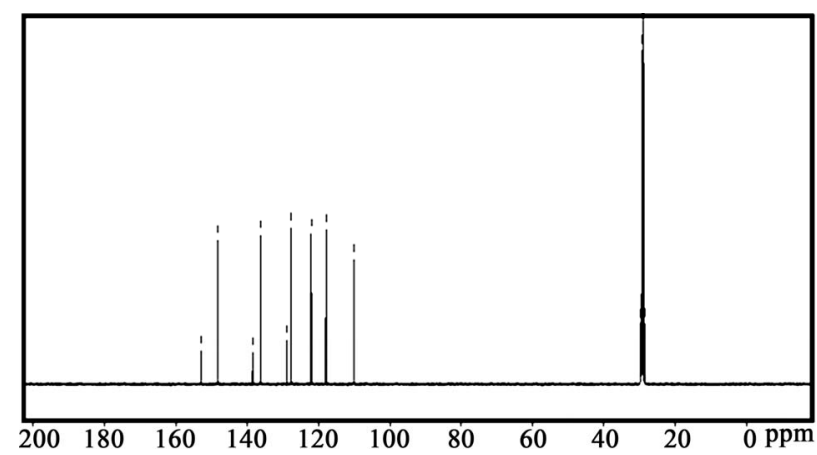

Figure $9 .{ }^{13} \mathrm{C}$ NMR spectrum of as grown pure 8-HQ single crystal.

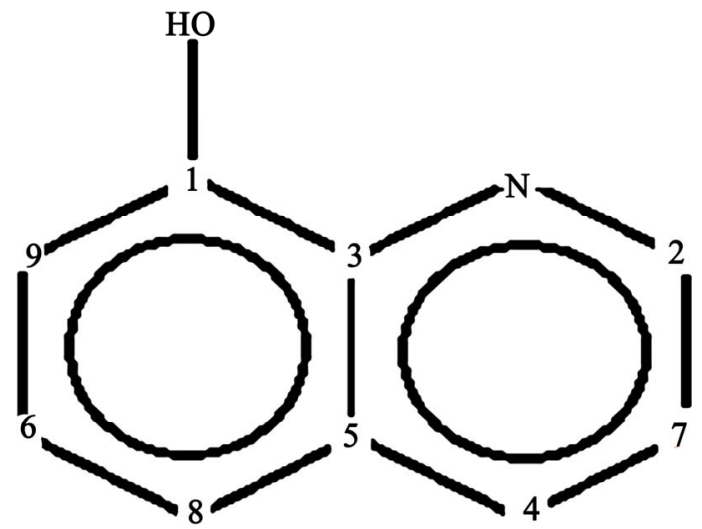

Figure 10. Structure of 8-HQ single crystal. 


\section{NLO Test}

The NLO property of pure 8-HQ and B8-HQ were measured by Kurtz Perry's NLO test. ANd-YAG laser beam of wavelength $1064 \mathrm{~nm}$ of pulse width $8 \mathrm{~ns}$ and repetition rate of $10 \mathrm{~Hz}$ was made to incident on the sample. Second harmonic radiation generated by $8-\mathrm{HQ}$ and B8HQ were focused by a lens and collected by a photomultiplier tube. In our study, KDP was taken as a reference crystal. The output power intensity of pure 8 -HQ was found to be 0.75 times that of KDP [4] and B8-HQ was found to be 0.85 times that of KDP single crystal.

\section{Conclusion}

Single crystals of pure 8-HQ and B8-HQ were grown successfully by slow evaporation technique at room temperature. Defect free, optically transparent single crystals of 8-HQ and B8-HQ were harvested within a month. The non-Centro-symmetric nature of the crystals was confirmed by XRD. The various functional groups assigned to 8-HQ and B8-HQ were confirmed by FTIR spectral analysis. The UV-Vis-NIR studies show that the cut off wavelength of 8-HQ is at $350 \mathrm{~nm}$ and that of B8$\mathrm{HQ}$ is at $356 \mathrm{~nm}$. The protons and the carbon bonded network in these samples were elucidated by ${ }^{1} \mathrm{H}$ and ${ }^{13} \mathrm{C}$ NMR spectral analysis. Studies pertaining to TG/DTA, SEM, Dielectric and Photoconductivity are in progress.

\section{Acknowledgements}

One of the authors (M. J. Jarald Brigit Gilda) is pleased to acknowledge Prof. I. Sebasdiyar, Head of the department of physics, St. Xavier's college, Palayamkottai and Dr. Maniysundar, Principal, Ponjesly College of Engineering, for their constant help, support and encouragement.

\section{REFERENCES}

[1] S. Palanisamy and O. N. Balasundaram, "Growth, Optical and Mechanical Properties of Alanine Sodium Nitrate
(ASN)," Rasayan Journal of Chemistry, Vol. 1, No. 4, 2008, pp. 782-787.

[2] C. Sekar and R. Parimala Devi, "Effect of Silver Nitrate $\left(\mathrm{AgNO}_{3}\right)$ on the Growth, Optical, Spectral, Thermal and Mechanical Properties of $\gamma$-Glycine Single Crystal," Journal of Optoelectronics and Biomedical Materials, Vol. 1, No. 2, 2009, pp. 215-225.

[3] M. Rajasekaran, P. Anbusrinivasan and S. C. Mojumdar, "Growth, Spectral and Thermal Characterization of 8-HydroxyQuinoline," Journal of Thermal Analysis and Calorimetry, Vol. 100, No. 3, 2010, pp. 827-830. doi10.1007/s10973-010-0761-5

[4] K. Aravinth, G. Anandha Babu and P. Ramasamy, "Growth of $<201>8$-HydroxyQuinoline Organic Crystal by Czochralski Method and Its Characterizations," Journal of Thermal Analysis and Calorimetry, 2011, 7 p.

[5] E. M. Filip, I. V. Humelnicu and C. I. Ghirve, "Some Aspects of 8-HydroxyQuinoline in Solvents," Acta Chemica Iasi, Vol. 17, 2009, pp. 85-96.

[6] C.-J. Mao, D.-C. Wang, H.-C. Pan and J.-J. Zhu, "Sonochemical Fabrication of 8-HydroxyQuinoline Aluminum $\left(\mathrm{Alq}_{3}\right)$ Nanoflowers with High Electrogenerated Chemiluminescence," Ultrasonics Sonochemistry, Vol. 18, No. 2, 2011, pp. 473-476.

[7] J. M. S. Skakle, J. L. Wardell and S. M. S. V. Wardell, "Formation of Ladders from R44(8) and R66(12) Rings in 8-Hydroxyquinoliniumchloride Monohydrate: Comparisons with the Supramolecular Arrangements in Related Salts," Acta Crystallographica Section C Crystal Structure Communications, Vol. 62, 2006, pp. 312-314.

[8] J. G. Mahakhode, B. M. Bahirwar, S. J. Dhoble and S. V. Moharil, "Tunable Photoluminescence from Tris (8-Hydroxyquinoline) Aluminum $\left(\mathrm{Alq}_{3}\right)$," Proceedings of Asian Symposium on Information Display, New Delhi, 8-12 October 2006, pp. 237-239.

[9] I. M. Khan, N. Singh and A. Ahmad, "Spectroscopic Studies of Multiple Charge Transfer Complexes of 8-HydroxyQuinoline with $\pi$-Acceptor P-Nitrophenol in Different Solvents at Room Temperature," Canadian Journal of Analytical sciences and Spectroscopy, Vol. 54, No. 1, 2009, pp. 31-37. 